Documentation et bibliothèques

DOCUMENTATION BIBLIOTHEQUES

\title{
Les besoins de personnel professionnel dans les bibliothèques publiques du Québec
}

\section{Réal Messier}

Volume 20, numéro 4, décembre 1974

URI : https://id.erudit.org/iderudit/1055657ar

DOI : https://doi.org/10.7202/1055657ar

Aller au sommaire du numéro

Éditeur(s)

Association pour l'avancement des sciences et des techniques de la documentation (ASTED)

ISSN

0315-2340 (imprimé)

2291-8949 (numérique)

Découvrir la revue

Citer cet article

Messier, R. (1974). Les besoins de personnel professionnel dans les bibliothèques publiques du Québec. Documentation et bibliothèques, 20(4),

179-182. https://doi.org/10.7202/1055657ar
Résumé de l'article

Après avoir examiné la situation actuelle dans les bibliothèques publiques du Québec, au point de vue du nombre des bibliothécaires professionnels, l'auteur tente d'évaluer les besoins actuels et futurs tout en tenant compte de la main-d'oeuvre disponible et des facteurs socio-économiques qui influencent les nouveaux diplômés dans leur choix.
Tous droits réservés (c) Association pour l'avancement des sciences et des techniques de la documentation (ASTED), 1974
Ce document est protégé par la loi sur le droit d'auteur. L'utilisation des services d'Érudit (y compris la reproduction) est assujettie à sa politique d'utilisation que vous pouvez consulter en ligne.

https://apropos.erudit.org/fr/usagers/politique-dutilisation/ 


\title{
Les besoins de personnel profes- sionnel dans les bibliothèques publiques du Québec
}

\author{
Réal Messier \\ Service des bibliothèques publiques \\ Ministère des Affaires culturelles \\ Québec
}

\begin{abstract}
Après avoir examiné la situation actuelle dans les bibliothèques publiques du Québec, au point de vue du nombre des bibliothécaires professionnels, l'auteur tente d'évaluer les besoins actuels et futurs tout en tenant compte de la main-d'oeuvre disponible et des facteurs socio-économiques qui influencent les nouveaux diplômés dans leur choix.
\end{abstract}

Dans un article à caractère sensationnel paru dans Le Devoir (27 mars 1972) et repris dans le Bulletin de I'ACBLF (septembre 1972), Emilien Tremblay écrivait:

"Seulement 133 bibliothécaires professionnels oeuvrent dans les bibliothèques publiques (...) En fait les bibliothécaires diplômés ne viendront aux bibliothèques publiques que lorsque les salaires offerts et les conditions de travail seront sensiblement les mêmes que dans les autres secteurs. Si le Directeur du Service des bibliothèques publiques s'en donnait la peine, il trouverait tout le personnel pour ouvrir cinq BCP dès I'automne 1972." 1

Daniel Reicher, reprenant le sujet de la formation du personnel pour les bibliothèques québécoises, affirmait:

“Il y a une forte probabilité que le nombre de nouveaux postes dans les bibliothèques publiques québécoises soit à peu près nul d'ici 1991, à moins que les municipalités ne changent fondamentalement leur politique budgétaire". 2

Face à ces affirmations, nous voudrions examiner la situation actuelle des bibliothécaires professionnels dans les bibliothèques publiques du Québec et voir quels sont les besoins immédiats et futurs, tout en tenant compte du nombre d'étudiants qui obtiennent un diplôme des écoles de bibliothéconomie et des facteurs qui peuvent affecter la disponibilité de cette main-d'oeuvre spécialisée.

1. Emilien Tremblay, "La grande misère des bibliothèques publiques", Bulletin de I'ACBLF, vol. XVIII, no 3 (septembre 1972), 188.

2. Daniel Reicher, "La formation du bibliothécaire pour le milieu québécois", Documentation et bibliothèques, vol. XIX, no 3 (septembre 1973), 109.
Lorsque nous parlons du bibliothécaire professionnel, il s'agit du candidat qui a complété un cours universitaire en bibliothéconomie, qu'il soit membre ou non de la Corporation des bibliothécaires professionnels du Québec.

\section{Situation actuelle}

En 1971-72, on retrouvait 122 bibliothécaires professionnels dans les bibliothèques publiques du Québec et 131 l'année suivante. De ce nombre, la majorité oeuvre dans la région métropolitaine.

Si nous examinons brièvement la situation et abordons la répartition par région administrative, l'éclairage est tout différent. On ne retrouve aucun professionnel dans les régions administratives 01, 09 et 10, c'est-à-dire le Bas St-Laurent, la Côte-Nord et le Nouveau-Québec; un seul bibliothécaire dans la région 08 qui recouvre tout le territoire du Nord-Ouest québécois et seulement 2 dans l'Estrie (05); 6 par contre dans l'Outaouais (07) et 7 dans les régions de Québec (03) et de la Mauricie (04). La région métropolitaine (06), fortement desservie, emploie 96 bibliothécaires professionnels, soit $78 \%$ d'entre eux.

En 1972-73, le nombre de professionnels est passé à 131 et tous se sont ajoutés au groupe de la région métropolitaine.

La population du Québec était, en 1973, selon Statistique Canada, d'environ 6,098,000 habitants, ce qui équivaut à 1 bibliothécaire professionnel pour 45,850 citoyens. Nous sommes donc très loin des normes suggérées par diverses associations professionnelles.

Chez nos voisins de l'Ontario, les bibliothèques publiques $y$ engageaient, en 1972, 826.9 bibliothécaires ou $\mathbf{1 . 1 2}$ professionnel par 
10,000 habitants $^{3}$. La comparaison devient plus que ridicule si nous regardons du côté des pays scandinaves où la bibliothèque publique est une tradition:

"As of April 1, 1970 there was one librarian for each 4,820 inh. working in the Danish public libraries or 2.07 librarians per 10,000 inhabitants." 4

\section{Besoins}

$\mathrm{Si}$ nous voulons atteindre l'excellence de I'Ontario, il faudrait, dans un premier temps, que le personnel professionnel affecté aux bibliothèques publiques soit de I'ordre de 610 bibliothécaires, donc à peu près cinq fois plus qu'actuellement.

Le Service de recherches et le Service des bibliothèques publiques du ministère des Affaires culturelles ont tenté d'évaluer ces besoins de bibliothécaires professionnels. ${ }^{5} \mathrm{En}$ prenant pour hypothèse qu'il fallait desservir toute la population québécoise (1971-72) et en retenant la moyenne des bibliothécaires professionnels per capita dans les provinces de Nouvelle-Ecosse, d'Ontario et de ColombieBritannique et en transposant cette moyenne de professionnels au Québec, on arrive à un besoin de 441 bibliothécaires.

Le tableau suivant illustre la situation actuelle et les besoins selon les hypothèses formulées. A noter que la région métropolitaine nécessiterait plus de $50 \%$ de ce total.
Régions administratives

$$
\begin{gathered}
\text { Situa- } \\
\text { tion } \\
1971-72
\end{gathered}
$$

Besoins pour desservir $100 \%$ en 1971-72

01 Bas St-LaurentGaspésie

02 Saguenay-Lac St-Jean

03 Québec

04 Mauricie

05 Estrie

06 Montréal

07 Outaouais

08 Nord-Ouest

09 Côte-Nord

10 Nouveau-Québec

\section{Total}

17

21

69

30

16

249

12

8

2

441
3. Ontario. Ministry of Colleges and Universities. Provincial Library Service, "Public libraries in Ontario Statistics for the year ending December 31, 1972"', Ontario Library Review, vol. 57, no. 4 (December 1973), 274.

4. Bbrge Sørensen, "The demand for librarians in Denmark 1970-1985", Scandinavian Public Library Quarterly, vol. 5, no. 1 (1972), 20.

5. Québec, Ministère des Affaires culturelles, Mémoire d'élément de programme: le développement d'un réseau de bibliothèques publiques au Québec, Québec, 1973, p. 37.
Quant aux effectifs futurs, ils ne eesseront de s'accentuer. On estime que la population du Québec en 1986 se situera entre 7.6 et 8.6 millions d'habitants. En supposant un faible taux de natalité et une immigration nette, on évalue la population à $7,633,000$ habitants. ${ }^{6} \mathrm{Si}$ nous appliquons la moyenne ontarienne actuelle qui est pourtant très réaliste, soit un professionnel pour 10,000 habitants, cela signifie qu'il faudrait 760 bibliothécaires d'ici une douzaine d'années.

Doit-on, comme Daniel Reicher, parler de saturation vers 1990? L'Ecole de bibliothéconomie est-elle en mesure de répondre à ce besoin du milieu? L'Ecole de bibliothéconomie, dans sa période de réorientation, devra se préoccuper de cette situation, dût-elle délaisser les secteurs universitaire et collégial et accentuer la formation de bibliothécaires pour le secteur public, qu'ils soient animateurs, bibliothécaires, administrateurs ou autres.

Les normes et les chiffres ne font que souligner une situation qui nous était déjà connue. Le marché existe, il s'agit de s'y infiltrer, de sensibiliser et de gagner les conseils municipaux à la cause des bibliothèques publiques.

Daniel Reicher formule un élément de solution à ce problème lorsqu'il ajoute: "à moins que les municipalités ne changent fondamentalement leur politique budgétaire." 7 La prochaine étape serait peut-être celle de la rédaction d'une politique provinciale municipale du développement des bibliothèques publiques.

Soit dit en passant, le projet de règlements des bibliothèques publiques insiste sur la responsabilité des municipalités dans le développement des bibliothèques publiques.

\section{Disponibilité}

Au-delà de la situation très inconfortable que connaissent les bibliothèques publiques québécoises, au-delà des normes, des besoins et des chiffres, une question demeure fondamentale: y a-t-il une main-d'oeuvre disponible?

Avant de jeter la pierre à qui que ce soit, il faudrait voir ce que l'on a fait pour attirer les nouveaux diplômés vers le secteur public, savoir pourquoi ils ne se sont pas orientés vers les bibliothèques publiques et s'interroger sur

6. Jacques Henripin et Jacques Légaré, Evolution démographique du Québec et de ses régions 1966-1986, Québec, PUL, 1969, p. 66.

7. Daniel Reicher, “La formation ... ", 109. 
I'infériorité des salaires et des conditions de travail qu'on rencontre dans ce secteur.

\section{Règlements}

Le premier texte des règlements des bibliothèques publiques mentionnait qu'une subvention de $\$ 1,000$ était accordée pour chaque bibliothécaire professionnel employé à plein temps; plus tard, un montant de $\$ 5,000$ était versé pour le bibliothécaire en chef. En 1972, un amendement à cet article exigeait que ce directeur soit membre de la Corporation des bibliothécaires professionnels du Québec.

$D$ 'autres articles de ce règlements stipulaient que les octrois ne pouvaient excéder $\$ 20,000$ pour les municipalités de moins de 40,000 habitants, $\$ 35,000$ pour les villes de 40,000 à 100,000 habitants et $\$ 60,000$ pour les autres, à I'exception de Montréal qui recevait $\$ 130,000$.

Nous en sommes au point où, avec ou sans bibliothécaire professionnel, $50 \%$ de nos bibliothèques publiques, grâce à leur effort local, reçoivent la subvention maximum.

Cet octroi de $\$ 5,000$ a été bénéfique entre 1965 et 1968 puisque le nombre de professionnels a doublé pour ensuite se stabiliser par la suite. Certaines municipalités qui ont bénéficié de cette subvention ont cru bon, lorsque le bibliothécaire quittait son poste, de ne pas retenir les services d'un bibliothécaire professionnel. II y a donc lieu de remettre en question cet article des règlements et, peut-être, d'imaginer une autre façon de s'assurer les services de bibliothécaires professionnels.

\section{Salaires}

A chaque année, Library Journal recense les salaires et les postes offerts aux diplômés des écoles de bibliothéconomie agréées par I'American Library Association.

Ainsi, y découvre-t-on qu'en 1973, les diplômés de l'université de Toronto ont reçu un traitement médian de $\$ 9,100$ tandis que ceux de Montréal recevaient $\$ 8,850$, ceux de $V$ ancouver $\$ 9,200$ et les diplômés de l'université Dalhousie, à Halifax, $\$ 8,565$. Toutefois, la médiane des salaires, pour l'ensemble du Canada, était de $\$ 9,100.8$

Les renseignements fournis par Library Journal nous laissent, en outre, clairement voir que les traitements accordés dans le secteur des bibliothèques publiques sont inférieurs à ceux

8. Carlyle J. Frarey \& Carol L. Learmont, "Placements and salaries 1973: not much change", Library Journal, vol. 99, no. 13 (July 1974), 1769. offerts par les bibliothèques spécialisées, les bibliothèques de collège et d'université et nettement inférieurs à ceux du secteur scolaire, tant au Canada qu'aux Etats-Unis.

Les directeurs des bibliothèques publiques recevaient, en moyenne, $\$ 11,115$ annuellement tandis que leurs collègues du secteur des collèges atteignaient \$12,230 par année (1972); par ailleurs, les commissions scolaires accordaient aux bibliothécaires un traitement annuel moyen de $\$ 9,236$, ceci excluant les coordonnateurs et le personnel de l'audio-visuel. La moyenne des salaires pour les bibliothécaires universitaires en 1972 au Québec était de \$10,712.

En plus de toutes ces données concernant les traitements, d'autres chiffres nous laissent songeur. Parmi les 26 finissants de l'université de Montréal en 1972, seulement 2 d'entre eux se sont orientés vers le secteur public, alors que 7 ou 27\% se dirigeaient vers les secteurs des collèges ou des universités. Du côté anglophone, par contre, les finissants de l'université de Toronto allaient surtout vers les bibliothèques publiques, soit 42 parmi les 97 diplômés, et 20 au secteur des collèges ou des universités. Vancouver rapporte 18 finissants au secteur public et 7 au secteur académique parmi ses 42 diplômés. L'énumération pourrait se poursuivre, mais I'on voit bien qu'il y a un malaise chez nous.

\section{Choix}

Laurent $\mathrm{G}$. Denis, dans sa thèse de doctorat soutenue à Rutgers en $1969^{\circ}$, a étudié les facteurs qui influencent les diplốmés des écoles de bibliothéconomie au Canada à opter pour le secteur public ou universitaire. Ces facteurs pouvaient souvent être d'ordre circonstanciel, c'est-à-dire l'expérience précédente dans un type de bibliothèque, les programmes offerts par les écoles, l'influence et le prestige du milieu, l'éducation et le statut socioéconomique du candidat. L'autre facteur retenu comme hypothèse était la personnalité du candidat.

Les conclusions de l'étude nous apprennent que ceux qui se dirigent vers le secteur académique le font parce qu'ils sont intéressés par la vie du milieu universitaire, le rôle de la bibliothèque dans l'éducation et aussi parce qu'ils sont familiers avec le milieu; tandis que ceux qui s'orientent vers le secteur public le font pour le besoin de servir et de rencontrer les gens, soit qu'ils aient été des usagers ou soit

9. Laurent G. Denis, Academic and public librarians in Canada: a study of the factors which influence graduates of Canadian library schools in making their first career decision in favor of academic or public libraries, New Brunswick, N.J., Rutgers University, 1969. 
qu'ils aient déjà travaillé dans une bibliothèque publique.

La personnalité des candidats explique quelque peu la situation de la main-d'oeuvre professionnelle dans les bibliothèques publiques du Québec.

Grâce à des tests ou à des formules standardisées et sans vouloir tracer un profil ou un portrait-robot, Laurent Denis caractérise ainsi les candidats de son échantillonnage qui s'orientent vers le secteur académique:

"The academic librarian prefers the arts to the sciences, enjoys human contact, has a good drive for achievement, is orderly, is unassuming, likes routine, and is suspicious of change. Furthermore, he is not a joiner (..) In short, he is like his reasons for becoming an academic librarian: serious, selfless, and a little bit pompous." 10

Quant à celui qui s'oriente vers le secteur public, il a tendance à être individualiste, suffisant mais bon et dévoué. Le bibliothécaire (homme) du secteur public n'offre rien de particulier, mais:

"... the Canadian female public librarians are greater achievers and are more aggressive. They are less respectful of authority, less orderly, more retiring, somewhat less dominant $(\ldots)$ In short, the Canadian male public librarian is not particularly noticeable person but his female counterpart shows distinguishing features which make her pleasant, unassuming, kind, reliable and dependable."11

Ces quelques caractéristiques nous font entrevoir l'image que projette le personnel de nos bibliothèques. II est évident que si nous voulons amener les gens à la bibliothèque, nous devrons faire disparaître quelques clichés ou archétypes qui ont identifié le personnage du "bibliothécaire."

Les informations révélées par Laurent Denis devraient amener le comité d'admission de l'Ecole de bibliothéconomie à sélectionner des éléments dynamiques, énergiques, engagés et ne plus accorder une importance primordiale au relevé de notes.

\section{En guise de ...}

Ces données illustrent l'ampleur et la diversité des problèmes que rencontrent les biblio- thèques publiques. Durant les années 60 , ces problèmes s'enfermaient dans un cercle vicieux. Aujourd'hui, malgré l'évolution et l'essor de notre réseau de bibliothèques publiques, le cercle demeure vicieux mais la circonférence s'atténue.

La formule ou la solution à ce problème ne viendrait donc pas uniquement du gouvernement du Québec. Dans un premier temps, ce milieu décisionnel doit développer des règlements appropriés à la situation municipale du Québec et, dans un second temps, amener, même par des mesures coercitives, les municipalités à assumer cette responsabilité de service public. Le milieu professionnel se doit également d'exercer des pressions afin de sensibiliser la population à ce besoin.

II y a certes lieu d'établir des consultations entre les écoles de formation et les projets ou politiques élaborés par le groupe de Québec, et ce dernier devrait rencontrer plus fréquemment les municipalités et leurs représentants.

Des études scientifiques et prospectives devraient être menées par les groupes impliqués afin d'établir les besoins et les mécanismes qui permettraient de remédier à cette situation.

Afin de s'infiltrer dans le milieu, les bibliothécaires devront adopter des attitudes plus dynamiques, plus combatives, afficher une présence au sein de la communauté et exercer des pressions auprès des autorités municipales afin qu'elles assument leurs responsabilités.

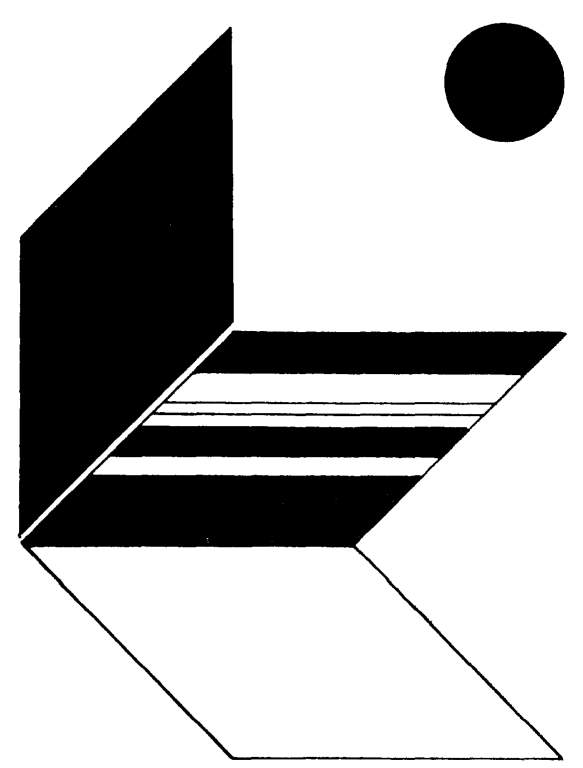

